\title{
Efecto antihelmíntico in vitro de extractos de plantas sobre larvas infectantes de nematodos gastrointestinales de rumiantes ${ }^{\#}$
}

\author{
In vitro anthelmintic effect of plant extracts against infective larvae \\ of ruminants gastrointestinal nematode parasites \\ FC Moreno $^{\mathrm{a}, \mathrm{b}, \mathrm{d}^{*}}$, IJ Gordon ${ }^{\mathrm{a}}$, AD Wright ${ }^{\mathrm{c}}$, MA Benvenutti ${ }^{\mathrm{a}, \mathrm{d}}$, CA Saumell ${ }^{\mathrm{b}}$ \\ ${ }^{a}$ CSIRO Davies Laboratory, University Drive, Townsville, Queensland, Australia. \\ ${ }^{\mathrm{b}}$ Facultad de Ciencias Veterinarias, U.N.C.P.B.A. Campus Pje., Arroyo Seco s/n, Tandil, Argentina. \\ ${ }^{\mathrm{c} A I M S}$ Australian Institute of Marine Science, Townsville, Queensland, Australia. \\ ${ }^{\mathrm{d} I N T A}$ EEA Cerro Azul, Cerro Azul, Misiones, Argentina.
}

\begin{abstract}
SUMMARY
With the purpose of studying the anthelmintic efficacy of some plant species presents in Queensland State, Australia, we tested in vitro the effect of plant extracts on infective larvae (L3) migration of Haemonchus placei, Cooperia sp., Haemonchus contortus and Trichostrongylus colubriformis. In general, plant extracts reduced the larval migration of Haemonchus placei and Cooperia sp. The most effective plants against Haemonchus placei and Cooperia sp. ( $\mathrm{P}<0.0001)$ were Allocasuarina torulosa, Neolitsea dealbata, Acacia holosericea, Acacia salicina, Callitris endlicheri and Casuarina cunninghamiana. Plants extracts were less effective on L3 migration of Haemonchus contortus and Trichostrongylus colubriformis. Callitris endlicheri, Casuarina cunninghamiana, Acacia farnesiana, Acacia holosericea and Acacia nilotica were the plant extracts that shown an important larval migration inhibition against $H$. contortus and Trichostrongylus colubriformis $(\mathrm{P}<0.0001)$. Callitris endlicheri was the plant that consistently inhibited the larval migration of every nematode species under study. These in vitro results suggest anthelmintic properties associate with some of the plant species we studied.
\end{abstract}

Palabras clave: evaluación in vitro, nematodos gastrointestinales, plantas, propiedades antihelmínticas.

Key words: in vitro test, gastrointestinal nematodes, plants species, anthelmintic properties.

\section{INTRODUCCIÓN}

Los parásitos nematodos gastrointestinales de rumiantes representan un serio problema a nivel mundial ya que afectan la productividad del hospedador causando reducciones en las tasas de crecimiento, fecundidad e incremento en la mortalidad. La parasitosis gastrointestinal es una de las enfermedades que mayor impacto económico ocasiona en los sistemas de producción de carne (Mcleod 1995, Fiel y Steffan 1994).

Hasta el presente, el principal modo de control de los parásitos del tracto digestivo ha estado basado en el tratamiento químico con antiparasitarios. Debido al creciente desarrollo de resistencia a los antiparasitarios por parte de los nematodos gastrointestinales (Jackson y Coop 2000) es necesario investigar estrategias alternativas al control farmacológico de las helmintiasis. A esto se suma la

Aceptado: 24.03.2010.

\# Fuente financiamiento: INTA-Instituto Nacional de Tecnología Agropecuaria, Argentina, CSIRO - Sustainable Ecosystem, Townsville, Australia.

* Pinto 399 (7000) Tandil, Argentina; fmoreno@ balcarce.inta.gov.ar. creciente demanda de limitar el uso de sustancias químicas en la producción animal para reducir los residuos de drogas en los productos animales (McKellar 1997, Waller y Thamsborg 2004).

La necesidad de encontrar alternativas para el control de los nematodos que puedan reducir el uso de antihelmínticos es reconocida internacionalmente. Varias opciones están siendo investigadas, dentro de las cuales se pueden citar el control biológico, usando hongos nematófagos (Larsen 1999, Larsen 2000), la producción de vacunas contra helmintos (Smith 1999, Vercruysse y col 2007), inmunonutrición (Houdijk y Athanasiadou 2003) y estudios genéticos tendientes a producir animales resistentes a helmintos (Gray 1997, Gasbarre y Miller 2000). También se han iniciado experimentos sobre la influencia de la suplementación proteica en la dieta, que podría mejorar la respuesta a la vacunación y en el desarrollo de animales inmunorresistentes (Coop y Holmes 1996). Otra estrategia alternativa para el control de parásitos gastrointestinales la constituye el uso de metabolitos secundarios de las plantas (MSP) (Athanasiadou y Kyriazakis 2004), con el objetivo no solo de encontrar nuevos compuestos químicos eficaces contra helmintos, sino que también por su aplicabilidad desde el punto de vista orgánico, ya que ellos podrían reemplazar el 
uso de químicos sintéticos corrientemente usados, lo cual puede proveer métodos ecológicos para el tratamiento de parásitos usando plantas con propiedades antihelmínticas.

Los test in vitro son un medio apropiado para evaluar especies de plantas debido a que son rápidos de realizar y económicos, comparados a los test in vivo. Aunque se ha encontrado que existe una correlación significativa entre los test in vitro e in vivo en estudios de eficacia de los antihelmínticos y resistencia de los parásitos a las drogas sintéticas (Geary y col 1999, Athanasiadou y Kyriazakis 2004), no es claro aún si una relación similar existe para los MSP (Athanasiadou y Kyriazakis 2004). Por ello, es importante que los resultados de los test in vitro sean siempre validados in vivo antes de hacer generalizaciones respecto a las propiedades antiparasitarias de los MSP (Athanasiadou y Kyriazakis 2004).

Debido a que el parásito adulto es el blanco principal de los MSP, sería deseable poder utilizarlos para determinar el potencial antiparasitario de los MSP. Sin embargo, los parásitos adultos no pueden ser mantenidos vivos fuera del hospedador por más de 24 horas, lo cual es inadecuado para los test in vitro. Por ello, los test in vitro se realizan usualmente con los estadios infectantes (L3) en lugar de adultos (Geary y col 1999, Athanasiadou y Kyriazakis 2004).

El test in vitro de inhibición de la migración larval con L3 ha sido ampliamente usado para estudiar la eficacia antiparasitaria de los MSP en Nueva Zelanda (Molan y col 2004, Molan y col 2000') y Francia (HounzangbeAdote y col 2005, Paolini y col 2004). El test de inhibición de la movilidad larval fue desarrollado por Wagland y col (1992) y modificado por Rabel y col (1994) y Paolini y col (2004). El uso de L3 para el test de migración parece ser apropiado para el estudio de la eficacia antiparasitaria de los MSP debido a que el primer contacto de los MSP con los parásitos se da cuando las L3 son consumidas junto con el forraje. A su vez, el test de inhibición de la migración de L3 tiene la ventaja sobre los test basados en la eclosión de huevos o en el desarrollo larval en que las L3 son fácilmente disponibles y almacenables y son relativamente simples de usar (Rabel y col 1994).

El objetivo del presente trabajo fue utilizar el test in vitro de inhibición de la migración larval para estudiar el efecto antihelmíntico de 24 extractos de plantas autóctonas de Australia con potenciales propiedades antiparasitarias, a tres concentraciones diferentes $(5,15$ y $30 \mathrm{mg} / \mathrm{ml})$, en la migración de L3 de nematodos que afectan al ganado bovino (Haemonchus placei y Cooperia sp.) y caprino (Haemonchus contortus y Trichostrongylus colubriformis).

\section{MATERIALES Y MÉTODOS}

\section{DISEÑO EXPERIMENTAL}

El trabajo experimental se llevó a cabo en el CSIRO Davies Laboratory, Townsville, Australia. Se realizó un experimento in vitro para evaluar el efecto de extractos de plantas australianas en la migración de L3 de nematodos que afectan al ganado bovino (Haemonchus placei y Cooperia sp.) y caprino (Haemonchus contortus y Trichostrongylus colubriformis).

\section{PLANTAS}

Para decidir las especies de plantas a evaluar, libros de etnobotánica en medicina tradicional humana o veterinaria y otras fuentes de información fueron consultados (Lassak y McCarthy 1983, Seigler 2002, Anderson 1993, WHO $1999^{1}$, Chittendon $1956^{2}$, Cribb y Cribb $1981^{3}$, Bennet-Jenkins y Bryant 1996, Moore 2005 comunicación personal, Grieve $1984^{2}$, Satrija y col $1994^{4}$, Athanasiadou y col $2000^{\mathrm{a}, \mathrm{b}}$, Athanasiadou y col 2001).

Como resultado, 24 especies de plantas con potencial antihelmíntico fueron identificadas: Acacia farnesiana, Acacia melanoxylon, Acacia salicina, Acacia holosericea, Acacia flavescens, Acacia leptostachya, Acacia nilotica, Eucalyptus tessellaris, Eucalyptus tereticornis, Eucalyptus platyphylla, Eucalyptus corymbia, Eucalyptus drepanophylla, Eucalyptus citriodora, Eucalyptus moluccana, Eucalyptus crebra, Casuarina cunninghamiana, Callitris endlicheri, Melaleuca leucodendra, Alphitonia excelsa, Erythrina variegata, Neolitsea dealbata, Allocasuarina torulosa, Euphorbia hirta y Panicum minutiflora. De las 24 especies seleccionadas, 19 fueron evaluadas en bovinos y 14 en caprinos. La selección de las plantas se basó en tres criterios: a) plantas conocidas previamente como activas en el control de parásitos internos en animales o humanos, b) plantas con alto contenido de MSP, no mencionadas como antiparasitarias pero con potencial antihelmíntico, c) especies que sean abundantes ya que las especies raras podrían ser irrelevantes para los animales en pastoreo.

\section{PREPARACIÓN DE LOS EXTRACTOS DE PLANTAS}

Hojas frescas de las especies de plantas a evaluar fueron colectadas en el estado de Queensland, Australia, durante los meses de septiembre y octubre y mantenidas en freezer $\left(-20^{\circ} \mathrm{C}\right)$ hasta su procesamiento. Aproximadamente $50 \mathrm{~g}$ de material correspondiente a cada una de las especies fue secado en liofilizador (freeze drier) y posteriormente molido a un milímetro de tamaño de partícula. Setenta y cinco mililitros de metanol fueron adicionados a tres gramos del material ya molido y dejado en agitación durante toda una noche para favorecer la extracción de los compuestos. Las muestras extraídas fueron filtradas en filtro de papel

http://www.genhealth.com/garlic.htm. fecha consulta: 08/08/2005 2 http://www.ibiblio.org/pfaf/cgi-bin/arr_ht ml?Melaleuca+hypericifolia\&CAN=LATIND. fecha consulta: 08/08/2005.

http://www.ibiblio.org/pfaf/cgi-bin/arr_ht ml?Callitris+endlicheri $\&$ CAN=COMIND. fecha consulta: 08/08/2005.

4 http://www.ansci.cornell.edu/plants/medicinal/papaya.html. fecha consulta: 08/08/2005 
Whatmann $\mathrm{N}^{\circ}$ 40. El filtrado fue luego liofilizado para obtener los polvos de extracto.

El polvo de los extractos de las plantas fue disuelto en buffer fosfato ( $0,1 \mathrm{M}$ fosfato, $0,05 \mathrm{M} \mathrm{NaCl} ; \mathrm{pH} 7,2)$ y diluido en serie previo a la incubación. Las diluciones evaluadas fueron 5,15 y $30 \mathrm{mg} / \mathrm{ml}$. Dichas diluciones fueron elegidas debido a que son comparables con las concentraciones usadas in vivo. Asumiendo que el contenido gastrointestinal de bovinos de destete de aproximadamente $200 \mathrm{~kg}$ de peso vivo es de aproximadamente 30 litros y sabiendo que el consumo de alimento es de aproximadamente $4 \mathrm{~kg}$ de materia seca por día, y que el máximo rendimiento de extracto es de aproximadamente $15 \%$ (Van Soest 1982), la máxima concentración de extracto de planta en el tracto gastrointestinal sería de aproximadamente $20 \mathrm{mg} / \mathrm{ml}$ (4 kg/día x 0,15/30 lts) cuando el animal consume solamente plantas ricas en MSP.

\section{PARÁSITOS}

Las L3 provenientes de cultivos puros de $H$. placei fueron suministradas por el Dr. Malcolm Knox, del CSIRO Livestock Industries FD McMaster Laboratory, Armidale, Australia; y las L3 provenientes de Cooperia sp. fueron suministradas por Novartis Animal Health, New South Wales, Australia, para realizar la primoinfección de los bovinos donadores de L3.

Dos terneros de destete libres de parásitos fueron infectados con una dosis única de $10.000 \mathrm{~L}_{3} \mathrm{H}$. placei y otros dos animales con una dosis única de $15.000 \mathrm{~L}_{3}$ Cooperia sp. Los cuatro animales se mantuvieron en corrales individuales en las instalaciones del CSIRO's Lansdown Research Station, Townsville, Australia. L3 de H. placei y Cooperia sp. se obtuvieron de cultivos fecales después de 28 días postinfección siguiendo el procedimiento de Lyndall-Murphy (1993). Aproximadamente $30 \mathrm{~g}$ de heces y un volumen similar de vermiculita fueron puestos en botellas de cultivo. Las heces fueron cultivadas para producir L3 solamente si el recuento de huevos de parásitos excedió los 200 huevos por gramo.

L3 provenientes de cultivos puros de H. contortus y T. colubriformis fueron suministradas en su totalidad para realizar el test in vitro por el Dr. Malcolm Knox, del CSIRO Livestock Industries FD McMaster Laboratory, Armidale, Australia, por lo que no se necesitó contar con animales dadores.

\section{TEST DE INHIBICIÓN DE LA MIGRACIÓN LARVAL}

Para determinar la eficacia antihelmíntica de los extractos de plantas se utilizó el test in vitro de inhibición de la migración larval desarrollado por Wagland y col (1992) y modificado por Rabel y col (1994). Se construyeron filtros con tubos de acrílico transparente de $20 \mathrm{~mm}$ de largo y $7 \mathrm{~mm}$ de diámetro interno a los que se les adhirió una malla de nylon de $20 \mu \mathrm{m}$ en uno de sus extremos. Se utilizaron tubos plásticos de $2 \mathrm{ml}$ a los cuales se le agregó $0,4 \mathrm{ml}$ de extracto diluido (dilución final 5,15 y $30 \mathrm{mg} / \mathrm{ml}$ ) y 150 a $200 \mathrm{~L} 3$ suspendidas en $0,1 \mathrm{ml}$ de solución acuosa. Se realizaron tres repeticiones para cada una de las distintas concentraciones de los extractos de plantas y un control negativo (L3 en buffer fosfato) se corrió en paralelo. Los tubos fueron incubados a $37^{\circ} \mathrm{C}$ por 16 horas. El contenido total de los tubos $(0,5 \mathrm{ml})$ fue luego transferido a los filtros localizados dentro de cámaras de acrílico de $1,9 \mathrm{~cm}$ de diámetro interno. Dichas cámaras con los filtros fueron incubadas a temperatura ambiente por 18 horas para permitir a las larvas migrar a través de los filtros dentro de la cámara. Luego, los filtros fueron removidos de las cámaras y el número total de larvas en las cámaras $\mathrm{y}$ en los filtros fueron contados.

\section{ANÁLISIS ESTADÍSTICO}

La inhibición de la migración larval (IML) se determinó usando la siguiente fórmula:

$$
\mathrm{IML}=\frac{\mathrm{A}-\mathrm{B}}{\mathrm{A}} \times 100
$$

Donde $\mathrm{A}=$ proporción de larvas migradas en el control y $\mathrm{B}=$ proporción de larvas migradas en los tratamientos

Para la variable porcentaje de migración larval fue realizado un análisis de variancia con un arreglo factorial de planta por concentración utilizando el procedimiento PROC GLM de SAS V.9.1.3 (SAS, Institute Inc., Cary, NC, USA). Para obtener el cumplimiento de los supuestos, la variable fue transformada con la función arcoseno. Como la interacción resultó significativa fue estudiado el comportamiento de las plantas dentro de cada concentración. Con el objeto de detectar las mejores combinaciones de planta y concentración fueron realizadas comparaciones múltiples con la media más pequeña (CMM) (Kuehl 2001). Esta metodología estadística permitió detectar subconjuntos conformados por extractos de plantas con porcentajes de inhibición larvaria similares dentro de cada una de las concentraciones evaluadas. Cuando se compararon todas las combinaciones de extractos de plantas y concentraciones, la CMM detectó un subgrupo combinación extractoconcentración con los mejores porcentajes de inhibición larvaria para cada especie parasitaria.

\section{RESULTADOS}

La interacción entre extractos de plantas y concentraciones fue significativa en cada uno de los géneros parasitarios evaluados $(\mathrm{P}<0,0001)$.

El porcentaje de migración de larvas de Haemonchus place $i$ en el tratamiento control con solo buffer fue de $89,66 \%$. A la concentración de $5 \mathrm{mg} / \mathrm{ml}$ fue detectado efecto significativo entre plantas $(\mathrm{P}<0,0001)$. En el cuadro 1 puede observarse que la CMM detectó un 
subconjunto de extractos que afectaron la migración larval, conformado por las plantas Eucalyptus tereticornis, Eucalyptus tessellaris, Eucalyptus crebra, Eucalyptus moluccana, Eucalyptus platyphylla, Acacia flavescens, Acacia holosericea, Acacia leptostachya, Acacia salicina, Allocasuarina torulosa, Casuarina cunninghamiana y Neolitsea dealbata, con porcentajes de migración menores al $81,6 \%$. A la concentración de $15 \mathrm{mg} / \mathrm{ml}$ el efecto de los extractos también fue estadísticamente significativo ( $\mathrm{P}<0,0001)$ y la CMM detectó un subconjunto conformado solamente por la planta Allocasuarina torulosa con un porcentaje de migración de $4,19 \%$. A la concentración de $30 \mathrm{mg} / \mathrm{ml}$ fue detectado efecto significativo entre plantas $(\mathrm{P}<0,0001)$ y la CMM detectó un subconjunto de extractos conformado por las plantas Acacia holosericea, Acacia salicina, Allocasuarina torulosa, Callitris endlicheri, Casuarina cunninghamiana y Neolitsea dealbata, con porcentajes de migración menores al $20,5 \%$. Cuando se compararon todas las combinaciones de plantas y concentraciones, la CMM detectó un grupo conformado por las plantas Acacia holosericea, Acacia salicina, Allocasuarina torulosa, Callitris endlicheri,
Casuarina cunninghamiana y Neolitsea dealbata, a la concentración de $30 \mathrm{mg} / \mathrm{ml}$, y la planta Allocasuarina torulosa a la concentración de $15 \mathrm{mg} / \mathrm{ml}$ con porcentajes de migración menores al $21 \%$.

Para Cooperia sp., el porcentaje de migración larval en el tratamiento control con solo buffer fue de $93,78 \%$. En el cuadro 2 se observa que a la concentración de $5 \mathrm{mg} / \mathrm{ml}$ fue detectado efecto significativo entre plantas $(\mathrm{P}=0,0002)$ y la CMM detectó un subconjunto de extractos conformado por las plantas Acacia farnesiana, Acacia salicina, Allocasuarina torulosa, Casuarina cunninghamiana y Neolitsea dealbata, con porcentajes de migración menores al $73 \%$. A la concentración de $15 \mathrm{mg} / \mathrm{ml}$ el efecto de las plantas fue estadísticamente significativo $(\mathrm{P}<0,0001)$ y la CMM detectó un subconjunto de extractos que afectaron la migración larval, conformado por las plantas Acacia salicina, Allocasuarina torulosa y Neolitsea dealbata, con porcentajes de migración menores al 16\%. A la concentración de $30 \mathrm{mg} / \mathrm{ml}$ también fue detectado efecto significativo entre plantas $(\mathrm{P}<0,0001)$ y la CMM detectó un subconjunto de extractos, conformado por las plantas Acacia holosericea, Acacia melanoxylon, Acacia salicina,

Cuadro 1. Porcentaje de migración de larvas infectivas de Haemonchus placei después de la incubación con extractos de plantas a 5,15 y $30 \mathrm{mg} / \mathrm{ml}(\mathrm{n}=3)$.

Percentage migration of infective larvae of Haemonchus placei after incubation with plant extracts at 5,15 y $30 \mathrm{mg} / \mathrm{ml}(\mathrm{n}=3)$.

\begin{tabular}{|c|c|c|c|c|c|c|}
\hline \multirow{2}{*}{$\begin{array}{l}\text { Extracto } \\
\text { de plantas }\end{array}$} & \multicolumn{2}{|c|}{$[5 \mathrm{mg} / \mathrm{ml}]$} & \multicolumn{2}{|c|}{$[15 \mathrm{mg} / \mathrm{ml}]$} & \multicolumn{2}{|c|}{$[30 \mathrm{mg} / \mathrm{ml}]$} \\
\hline & Media & $E E$ & Media & $E E$ & Media & $E E$ \\
\hline Eucalyptus citriodora & 81,92 & 1,49 & 79,95 & 1,99 & 79,88 & 0,93 \\
\hline Eucalyptus tereticornis & $77,04 *$ & 2,48 & 72,12 & 3,95 & 58,72 & 4,03 \\
\hline Eucalyptus tessellaris & $81,64 *$ & 3,13 & 79,11 & 2,17 & 68,95 & 5,06 \\
\hline Eucalyptus crebra & $72,43 *$ & 4,61 & 68,37 & 1,68 & 66,55 & 5,56 \\
\hline Eucalyptus moluccana & $81,09^{*}$ & 1,51 & 84,47 & 1,79 & 74,01 & 5,33 \\
\hline Eucalyptus platyphylla & $73,45^{*}$ & 2,28 & 63,89 & 4,42 & 65,16 & 4,43 \\
\hline Acacia farnesiana & 91,49 & 1,18 & 82,85 & 0,44 & 80,48 & 7,52 \\
\hline Acacia flavescens & $77,09 *$ & 4,33 & 71,21 & 6,31 & 68,03 & 6,45 \\
\hline Acacia holosericea & $62,87^{*}$ & 4,29 & 54,77 & 3,36 & $11,80 * \#$ & 8,98 \\
\hline Acacia leptostachya & $80,18^{*}$ & 1,48 & 45,96 & 7,48 & 34,83 & 5,78 \\
\hline Acacia melanoxylon & 83,89 & 2,14 & 64,79 & 5,83 & 51,30 & 7,56 \\
\hline Acacia salicina & $74,24 *$ & 2,60 & 42,79 & 8,81 & $17,32 * \#$ & 4,69 \\
\hline Allocasuarina torulosa & $61,28 *$ & 10,28 & 4,19 *\# & 2,09 & 1,07 *\# & 0,45 \\
\hline Alphitonia excelsa & 90,17 & 1,90 & 74,18 & 3,47 & 67,61 & 9,35 \\
\hline Callitris endlicheri & 83,64 & 1,45 & 47,90 & 1,26 & $9,62 * \#$ & 4,82 \\
\hline Casuarina cunninghamiana & $78,08^{*}$ & 10,69 & 59,39 & 7,14 & $7,36^{* \#}$ & 5,24 \\
\hline Erythrina variegata & 92,24 & 3,27 & 49,97 & 8,32 & 49,43 & 6,36 \\
\hline Melaleuca leucodendra & 86,95 & 2,79 & 78,00 & 2,66 & 80,54 & 1,48 \\
\hline Neolitsea dealbata & $68,09 *$ & 4,81 & 34,93 & 6,60 & $20,41 * \#$ & 4,67 \\
\hline
\end{tabular}

* Subconjuntos de extractos con menor porcentaje de migración, detectados por la CMM en cada una de las concentraciones.

\# Subconjunto de combinación extractos/concentración con menor porcentaje de migración, detectados por la CMM. 
Cuadro 2. Porcentaje de migración de larvas infectivas de Cooperia sp. después de la incubación con extractos de plantas a 5 , 15 y $30 \mathrm{mg} / \mathrm{ml}(\mathrm{n}=3)$.

Percentage migration of infective larvae of Cooperia sp. after incubation with plant extracts at 5,15 y $30 \mathrm{mg} / \mathrm{ml}(\mathrm{n}=3)$.

\begin{tabular}{|c|c|c|c|c|c|c|}
\hline \multirow{2}{*}{$\begin{array}{l}\text { Extracto } \\
\text { de plantas }\end{array}$} & \multicolumn{2}{|c|}{$[5 \mathrm{mg} / \mathrm{ml}]$} & \multicolumn{2}{|c|}{$[15 \mathrm{mg} / \mathrm{ml}]$} & \multicolumn{2}{|c|}{$[30 \mathrm{mg} / \mathrm{ml}]$} \\
\hline & Media & $E E$ & Media & $E E$ & Media & $E E$ \\
\hline Eucalyptus citriodora & 82,92 & 1,46 & 74,11 & 2,29 & 85,75 & 1,87 \\
\hline Eucalyptus tereticornis & 90,20 & 1,83 & 73,47 & 9,39 & 69,19 & 4,09 \\
\hline Eucalyptus tessellaris & 91,78 & 2,17 & 86,06 & 2,30 & 56,16 & 1,90 \\
\hline Eucalyptus crebra & 89,59 & 1,63 & 84,92 & 3,24 & 79,32 & 5,10 \\
\hline Eucalyptus moluccana & 92,06 & 1,73 & 87,34 & 1,06 & 50,71 & 2,59 \\
\hline Eucalyptus platyphylla & 77,44 & 1,60 & 53,59 & 8,02 & 47,55 & 8,66 \\
\hline Acacia farnesiana & $72,71^{*}$ & 8,70 & 83,17 & 6,28 & 90,32 & 0,48 \\
\hline Acacia flavescens & 81,01 & 6,54 & 72,52 & 5,43 & 66,54 & 9,51 \\
\hline Acacia holosericea & 86,60 & 2,42 & 40,46 & 6,37 & $15,46^{* \#}$ & 8,88 \\
\hline Acacia leptostachya & 88,06 & 4,26 & 84,71 & 8,09 & 58,60 & 12,97 \\
\hline Acacia melanoxylon & 84,71 & 3,51 & 75,94 & 12,27 & $29,74 * \#$ & 11,51 \\
\hline Acacia salicina & $68,27 *$ & 7,96 & 13,52 *\# & 1,35 & 0,41 *\# & 0,21 \\
\hline Allocasuarina torulosa & $38,93 *$ & 16,31 & $5,55^{* \#}$ & 1,52 & 2,30 *\# & 1,16 \\
\hline Alphitonia excelsa & 84,75 & 1,26 & 81,69 & 4,82 & 72,85 & 9,90 \\
\hline Callitris endlicheri & 78,53 & 3,30 & 42,47 & 6,27 & 0,39 *\# & 0,20 \\
\hline Casuarina cunninghamiana & $72,14^{*}$ & 9,54 & 55,74 & 5,64 & $20,49^{\#}$ & 11,83 \\
\hline Erythrina variegata & 85,88 & 4,52 & 86,16 & 2,22 & 70,98 & 3,21 \\
\hline Melaleuca leucodendra & 92,77 & 0,51 & 87,84 & 3,59 & 88,32 & 1,03 \\
\hline Neolitsea dealbata & $66,64 *$ & 13,71 & $15,69^{* \#}$ & 2,00 & $2,07 * \#$ & 0,82 \\
\hline
\end{tabular}

* Subconjuntos de extractos con menor porcentaje de migración, detectados por la CMM en cada una de las concentraciones.

\# Subconjunto de combinación extractos/concentración con menor porcentaje de migración, detectados por la CMM.

Allocasuarina torulosa, Callitris endlicheri y Neolitsea dealbata, con porcentajes de migración menores al 30\%. Cuando se compararon todas las combinaciones de plantas y concentraciones, la CMM detectó un grupo conformado por las plantas Acacia holosericea, Acacia melanoxylon, Acacia salicina, Allocasuarina torulosa, Callitris endlicheri, Casuarina cunninghamiana y Neolitsea dealbata, a la concentración de $30 \mathrm{mg} / \mathrm{ml}$, y las plantas Acacia salicina, Allocasuarina torulosa y Neolitsea dealbata a la concentración de $15 \mathrm{mg} / \mathrm{ml}$ con porcentajes de migración menores al $30 \%$.

El porcentaje de migración de larvas de Haemonchus contortus en el tratamiento control con solo buffer fue de $99,3 \%$. A las concentraciones de $5 \mathrm{mg} / \mathrm{ml}$ y $15 \mathrm{mg} / \mathrm{ml}$ no fue detectado efecto significativo entre plantas $(\mathrm{P}=0,3777)$ y $(\mathrm{P}=0,0529)$ respectivamente. Sin embargo, a la concentración de $30 \mathrm{mg} / \mathrm{ml}$ (cuadro 3) fue detectado efecto significativo entre plantas $(\mathrm{P}=0,0053)$ y la CMM detectó un subconjunto de extractos que afectaron la migración larval, conformado por las plantas Acacia holosericea, Acacia nilotica, Acacia farnesiana, Callitris endlicheri y Casuarina cunninghamiana, con porcentajes de migración menores al $88 \%$. Cuando se compararon todas las combinaciones de plantas y concentraciones, la CMM detectó un grupo conformado por las plantas Acacia holosericea, Acacia nilotica, Acacia farnesiana, Callitris endlicheri y Casuarina cunninghamiana a la concentración de $30 \mathrm{mg} / \mathrm{ml}$ y la planta Casuarina cunninghamiana a la concentración de $15 \mathrm{mg} / \mathrm{ml}$ con porcentajes de migración entre 64,14 y $89,83 \%$.

Para Trichostrongylus colubriformis el porcentaje de migración larval en el tratamiento control con solo buffer fue de $99,5 \%$. A la concentración de $5 \mathrm{mg} / \mathrm{ml}$ no fue detectado efecto significativo entre plantas $(\mathrm{P}=0,4129)$. En el cuadro 4 puede observarse que a la concentración de $15 \mathrm{mg} / \mathrm{ml}$ fue detectado efecto significativo entre plantas $(\mathrm{P}=0,0006)$ y la $\mathrm{CMM}$ detectó un subconjunto 
F MORENO Y COL

Cuadro 3. Porcentaje de migración de larvas infectivas de Haemonchus contortus después de la incubación con extractos de plantas a 5,15 y $30 \mathrm{mg} / \mathrm{ml}(\mathrm{n}=3)$.

Percentage migration of infective larvae of Haemonchus contortus after incubation with plant extracts at 5,15 y $30 \mathrm{mg} / \mathrm{ml}(\mathrm{n}=3)$.

\begin{tabular}{|c|c|c|c|c|c|c|}
\hline \multirow{2}{*}{$\begin{array}{l}\text { Extracto } \\
\text { de plantas }\end{array}$} & \multicolumn{2}{|c|}{$[5 \mathrm{mg} / \mathrm{ml}]$} & \multicolumn{2}{|c|}{$[15 \mathrm{mg} / \mathrm{ml}]$} & \multicolumn{2}{|c|}{$[30 \mathrm{mg} / \mathrm{ml}]$} \\
\hline & Media & $E E$ & Media & $E E$ & Media & $E E$ \\
\hline Acacia holosericea & 97,41 & 2,59 & 98,28 & 0,84 & 83,49 *\# & 1,47 \\
\hline Acacia melanoxylon & 96,34 & 1,54 & 93,07 & 0,41 & 98,87 & 0,05 \\
\hline Acacia nilotica & 100 & 0 & 96,60 & 0,05 & $77,12^{\text {*\# }}$ & 5,41 \\
\hline Acacia salicina & 98,90 & 0,65 & 99,78 & 0,23 & 97,29 & 0,98 \\
\hline Acacia farnesiana & 98,78 & 0,67 & 98,42 & 0,58 & 87,79 *\# & 0,45 \\
\hline Callitris endlicheri & 98,32 & 0,25 & 96,87 & 2,20 & $64,14^{* \#}$ & 23,57 \\
\hline Casuarina cunninghamiana & 96,98 & 0,01 & $89,83^{\#}$ & 8,13 & 77,89 *\# & 16,70 \\
\hline Eucalyptus drepanophylla & 98,01 & 1,11 & 97,75 & 0,72 & 96,63 & 0,97 \\
\hline Eucalyptus platyphylla & 99,02 & 0,39 & 99,76 & 0,25 & 98,57 & 0,01 \\
\hline Eucalyptus tereticornis & 98,03 & 1,98 & 99,34 & 0,67 & 98,64 & 0,40 \\
\hline Eucalyptus tessellaris & 99,57 & 0 & 99,27 & 0,25 & 99,34 & 0,17 \\
\hline Eucalyptus corymbia & 97,09 & 1,23 & 99,78 & 0,23 & 99,77 & 0,23 \\
\hline Euphorbia hirta & 98,90 & 0,62 & 99,44 & 0,04 & 98,54 & 0,15 \\
\hline Panicum minutiflora & 95,91 & 1,36 & 96,41 & 2,11 & 98,44 & 0,57 \\
\hline
\end{tabular}

* Subconjuntos de extractos con menor porcentaje de migración, detectados por la CMM en cada una de las concentraciones.

\# Subconjunto de combinación extractos/concentración con menor porcentaje de migración, detectados por la CMM.

Cuadro 4. Porcentaje de migración de larvas infectivas de Trichostrongylus colubriformis después de la incubación con extractos de plantas a 5,15 y $30 \mathrm{mg} / \mathrm{ml}(\mathrm{n}=3)$.

$(\mathrm{n}=3)$.

Percentage migration of infective larvae of Trichostrongylus colubriformis after incubation with plant extracts at 5,15 y $30 \mathrm{mg} / \mathrm{ml}$

\begin{tabular}{|c|c|c|c|c|c|c|}
\hline \multirow{2}{*}{$\begin{array}{l}\text { Extracto } \\
\text { de plantas }\end{array}$} & \multicolumn{2}{|c|}{$[5 \mathrm{mg} / \mathrm{ml}]$} & \multicolumn{2}{|c|}{$[15 \mathrm{mg} / \mathrm{ml}]$} & \multicolumn{2}{|c|}{$[30 \mathrm{mg} / \mathrm{ml}]$} \\
\hline & Media & $E E$ & Media & $E E$ & Media & $E E$ \\
\hline Acacia holosericea & 99,14 & 0,05 & 99,40 & 0,16 & 98,51 & 1,49 \\
\hline Acacia melanoxylon & 99,16 & 0,45 & 99,22 & 0,02 & 98,27 & 0,49 \\
\hline Acacia nilotica & 99,09 & 0,91 & $97,22^{*}$ & 0,89 & 87,12 & 0,65 \\
\hline Acacia salicina & 100 & 0 & 100 & 0 & 97,65 & 1,51 \\
\hline Acacia farnesiana & 99,79 & 0,21 & 99,39 & 0,62 & 100 & 0 \\
\hline Callitris endlicheri & 99,17 & 0,04 & $92,96 *$ & 2,72 & $21,55^{* \#}$ & 14,96 \\
\hline Casuarina cunninghamiana & 97,55 & 1,06 & 100 & 0 & 86,86 & 8,58 \\
\hline Eucalyptus drepanophylla & 98,81 & 0,24 & 98,34 & 1,23 & 97,71 & 0,97 \\
\hline Eucalyptus platyphylla & 99,41 & 0,59 & 99,60 & 0,02 & 97,79 & 1,74 \\
\hline Eucalyptus tereticornis & 97,90 & 0,15 & 98,49 & 0,27 & 98,12 & 0,56 \\
\hline Eucalyptus tessellaris & 99,14 & 0,01 & 99,45 & 0,15 & 99,37 & 0,28 \\
\hline Eucalyptus corymbia & 99,57 & 0 & 100 & 0 & 100 & 0 \\
\hline Euphorbia hirta & 98,15 & 1,85 & 98,54 & 0,61 & 97,85 & 1,70 \\
\hline Panicum minutiflora & 99,50 & 0,08 & 98,13 & 0,61 & 97,03 & 1,68 \\
\hline
\end{tabular}

* Subconjuntos de extractos con menor porcentaje de migración, detectados por la CMM en cada una de las concentraciones

\# Subconjunto de combinación extractos/concentración con menor porcentaje de migración, detectados por la CMM. 
de extractos conformado por las plantas Acacia nilotica y Callitris endlicheri, con porcentajes de migración de $97,22 \%$ y $92,96 \%$, respectivamente. A la concentración de $30 \mathrm{mg} / \mathrm{ml}$ fue detectado efecto significativo entre plantas $(\mathrm{P}<0,0001)$ y la CMM detectó un subconjunto conformado solamente por la planta Callitris endlicheri con un porcentaje de migración de $21,55 \%$. Cuando se compararon todas las combinaciones de plantas y concentraciones, la CMM detectó solo la planta Callitris endlicheri a la concentración de $30 \mathrm{mg} / \mathrm{ml}$ como diferente del resto de los extractos.

\section{DISCUSIÓN}

Las especies de plantas estudiadas en el presente trabajo se eligieron debido a que fueron previamente nombradas en fuentes de etnobotánica como activas en el control de parásitos en animales o humanos e identificadas previamente por su alto contenido en MSP con potencial antiparasitario.

Para H. placei la CMM nos permitió detectar subconjuntos de extractos de plantas que causaron bajos porcentajes de migración dentro de cada una de las concentraciones evaluadas. La mayoría de las extractos analizados afectaron significativamente $(\mathrm{P}<0,0001)$ la migración de larvas a la concentración de $5 \mathrm{mg} / \mathrm{ml}$, pero los niveles de inhibición de la migración larval (IML) de entre 8 y $28 \%$ no fueron relevantes, mientras que a la concentración de $15 \mathrm{mg} / \mathrm{ml}$ el extracto de Allocasuarina torulosa logró una reducción importante de la migración con niveles de IML mayores a 85\%. Los extractos de Acacia holosericea, Acacia salicina, Allocasuarina torulosa, Callitris endlicheri, Casuarina cunninghamiana y Neolitsea dealbata, a la concentración de $30 \mathrm{mg} / \mathrm{ml}$ causaron una reducción de la migración con niveles de IML de 69\%.

Para Cooperia sp. la CMM detectó subconjuntos de extractos de plantas que causaron porcentajes de migración variables. A la concentración de $5 \mathrm{mg} / \mathrm{ml}$ los extractos lograron IML en un $22 \%$, mientras que a la concentración de $15 \mathrm{mg} / \mathrm{ml}$ y $30 \mathrm{mg} / \mathrm{ml}$ los niveles de IML llegaron a 78 y $64 \%$ respectivamente. Las especies de plantas que resultaron más efectivas en reducir significativamente la migración de larvas de H. placei y Cooperia sp. con porcentajes menores a $21 \%$ y $30 \%$ respectivamente fueron: Acacia holosericea, Acacia salicina, Allocasuarina torulosa, Callitris endlicheri, Casuarina cunninghamiana y Neolitsea dealbata (cuadro 1). Allocasuarina torulosa fue una de las especies que redujo la migración de larvas en ambas especies de parásitos a las diferentes concentraciones evaluadas. Acacia salicina, Allocasuarina torulosa y Neolitsea dealbata fueron las especies de plantas detectadas por el método CMM que demostraron una significativa capacidad de inhibición de la migración en L3 de Cooperia sp. a las tres concentraciones estudiadas.

Para el caso de las L3 de H. contortus y $T$. colubriformis, pudo observarse un menor efecto de los extractos vegetales evaluados, lo que se vio reflejado en los altos porcentajes de migración larval. Para $H$. contortus, solo a la concentración de $30 \mathrm{mg} / \mathrm{ml}$ pudieron detectarse extractos de plantas que redujeron la migración larval con bajos índices de IML (11\%), mientras que a la concentración de $15 \mathrm{mg} / \mathrm{ml}$ un solo extracto redujo escasamente la migración larval con un IML de 3\%. Estos valores demuestran una baja efectividad de los extractos, lo que se traduce en una reducida capacidad de inhibición de la migración de L3 de $H$. contortus a las tres concentraciones estudiadas

En el caso de T. colubriformis, si bien a la concentración de $15 \mathrm{mg} / \mathrm{ml}$ los niveles de IML (2,5\%) no fueron relevantes, a la concentración de $30 \mathrm{mg} / \mathrm{ml}$ el extracto de Callitris endlicheri logró una reducción importante de la migración con niveles de IML mayores a 78\%. Callitris endlicheri fue la única especie de planta detectada por el método CMM que mostró una significativa capacidad de inhibición de la migración larval.

Callitris endlicheri es una de las especies de plantas que redujo la migración de larvas en todas las especies de nematodos evaluadas en el presente estudio. Estos resultados concuerdan con los antecedentes que muestran que esta especie de planta fue previamente identificada por su alto contenido en taninos y por su efecto antihelmíntico en equinos (Lassak y McCarthy 1983, Chittendon 19565, Cribb y Cribb 19813).

Por su parte, Eucalyptus grandis es conocido por su efecto antihelmíntico en caprinos (Bennet-Jenkins y Bryant 1996). Sin embargo, en el presente estudio las especies de Eucalyptus evaluadas tuvieron un efecto leve en larvas de H. placei y Cooperia $s p$. y no afectaron la migración de larvas de $H$. contortus y $T$. colubriformis.

En general, las especies de Acacia (Seigler 2002, Anderson 1993) y Eucalyptus $^{6}$ son conocidas por su alto contenido en MSP siendo particularmente ricas en taninos. En el presente estudio las especies de Acacia presentaron una mayor actividad antihelmíntica que las especies de Eucalyptus. Por ejemplo, Acacia holosericea y Acacia salicina estuvieron dentro de las especies que mostraron tener actividad para H. placei y Cooperia sp., mientras que ningún Eucalyptus se encontró dentro de este grupo. Algo similar ocurrió en $H$. contortus y T. colubriformis donde Acacia holosericea, Acacia nilotica, Acacia farnesiana fueron las especies más activas, pero ningún Eucalyptus se encontró dentro de este grupo.

Existe muy poca información acerca de la actividad antiparasitaria de las especies de plantas evaluadas en el presente estudio. A su vez, la poca información disponible en general no es clara. Es necesario que estos resultados generados in vitro sean validados con estudios in vivo. De

http://www.ibiblio.org/pfaf/cgi-bin/arr_ht ml?Callitris+endlicheri \&CAN=LATIND. fecha consulta: 08/08/2005.

6 Moore B. 2005. Senior Research Associate. School of Tropical Biology. James Cook University (comunicación personal). 
hecho, algunos estudios encontraron una buena relación entre los resultados in vitro e in vivo, por ejemplo Molan y col $\left(2000^{\mathrm{a}, \mathrm{b}}\right)$ encontraron que los taninos condensados purificados de varias especies vegetales redujeron la movilidad y migración de L 3 de nematodos y estos resultados estuvieron de acuerdo con los obtenidos en estudios in vivo en rumiantes (Athanasiadou y col 2000a, b , Paolini $\mathrm{y} \operatorname{col} 2003^{\mathrm{a}, \mathrm{b}}$ ). Sin embargo, se requiere prudencia en la extrapolación de los resultados in vitro a condiciones in vivo debido a que los componentes químicos de las plantas pueden ser modificados luego de pasar por el tracto digestivo. Esto es particularmente cierto en el ambiente ruminal que afecta a los taninos por degradación bacteriana (Makkar 2003). Sumado a ello deben tenerse presente los mecanismos adaptativos especiales desarrollados por los animales. La presencia de Streptococcus caprinus en el rumen de caprinos y la secreción de saliva rica en prolina son las principales adaptaciones experimentadas por los caprinos, en los cuales plantas ricas en taninos son parte de su dieta (Landau 2000). Por otra parte, la toxicidad de los taninos para los animales no puede ser evaluada in vitro. Es ampliamente conocido que bajas a moderadas concentraciones de taninos (3-6\% MS) mayoritariamente tuvieron efectos positivos en la fisiología del hospedador, crecimiento y producción de lana y leche (Min y col 2003), mientras que altas concentraciones (7-8\% MS) reducen la ganancia de peso y productividad de los animales e incluso pueden causarle la muerte (Waghorn y McNabb 2003). Por ello, una vez identificadas las especies de plantas con mayor potencial antihelmíntico a través de estudios in vitro, es necesario validar dichos resultados con estudios in vivo.

Se concluye que, en general, los extractos de plantas evaluados redujeron la migración de larvas de $H$. placei y Cooperia $s p$. Las plantas que mostraron una mayor inhibición de la migración larval contra estas especies de nematodos fueron Allocasuarina torulosa, Neolitsea dealbata, Acacia holosericea, Acacia salicina, Callitris endlicheri y Casuarina cunninghamiana.

Los extractos fueron menos efectivos en inhibir la migración de larvas de $H$. contortus y $T$. colubriformis. Las plantas que mostraron una mayor inhibición de la migración larval contra esta especies de nematodos fueron Callitris endlicheri, Casuarina cunninghamiana, Acacia farnesiana, Acacia holosericea y Acacia nilotica.

Callitris endlicheri fue la especie que inhibió la migración de todas las especies de nematodos estudiadas.

Estos resultados in vitro sugieren la existencia de propiedades antihelmínticas asociadas con algunas de las especies de plantas evaluadas. Para confirmar estos resultados in vitro es necesario conducir estudios in vivo con dichas plantas.

\section{RESUMEN}

Con el objeto de estudiar la capacidad antihelmíntica de algunas especies de plantas presentes en el Estado de Queensland, Australia, se evaluó el efecto in vitro de extractos de hojas de plantas en la migración de larvas infectantes (L3) de Haemonchus placei, Cooperia sp., Haemonchus contortus y Trichostrongylus colubriformis. En general, los extractos de plantas redujeron la migración de larvas de H. placei y Cooperia sp. Las plantas con mayor actividad antihelmíntica contra estas especies de nematodos fueron Allocasuarina torulosa, Neolitsea dealbata, Acacia holosericea, Acacia salicina, Callitris endlicheri y Casuarina cunninghamiana $(\mathrm{P}<0,0001)$. Los extractos fueron menos efectivos en inhibir la migración de larvas de $H$. contortus y $T$. colubriformis. Las plantas que mostraron una mayor inhibición de la migración larval contra estas especies de nematodos fueron Callitris endlicheri, Casuarina cunninghamiana, Acacia farnesiana, Acacia holosericea y Acacia nilotica $(\mathrm{P}<0,0001)$. Callitris endlicheri fue la especie que más consistentemente inhibió la migración de todas las especies de nematodos estudiadas. Estos resultados in vitro sugieren la existencia de propiedades antihelmínticas asociadas con algunas de las especies de plantas evaluadas.

\section{AGRADECIMIENTOS}

Los autores agradecen el apoyo técnico y económico del CSIRO Sustainable Ecosystem, Townsville, Australia, y del INTA, Argentina, para la realización de este trabajo. Un especial agradecimiento a Edgardo Rodríguez y Juan Passucci de la Facultad de Ciencias Veterinarias de la UNCPBA, por su ayuda en el análisis estadístico de los datos.

\section{REFERENCIAS}

Anderson ER. 1993. Plants of central Queensland: their identification and uses. Eric Anderson Dept. of Primary Industries, Brisbane, Qld., Australia.

Athanasiadou S, I Kyriazakis, F Jackson, RL Coop. 2000a . Consequences of long-term feeding with condensed tannins on sheep parasited with Trichostrongylus colubriformis. Int J Parasitol 30, 1025-1033.

Athanasiadou S, I Kyriazakis, F Jackson, RL Coop. 2000 . Effects of short-term exposure to condensed tannins on adult Trichostrongylus colubriformis. Vet Rec 146, 728-732.

Athanasiadou S, I Kyriazakis, F Jackson, RL Coop. 2001. Direct anthelmintic effects of condensed tannins towards different gastrointestinal nematodes of sheep: in vitro and in vivo studies. Vet Parasitol 99, 205-219.

Athanasiadou S, I Kyriazakis. 2004. Plant secondary metabolites: antiparasitic effects and their role in ruminant production systems. $P$ Nutr Soc 63, 631-639.

Bennet-Jenkins E, C Bryant. 1996. Novel sources of anthelmintics. Int J Parasitol 26, 937-947.

Coop RL, PH Holmes. 1996. Nutrition and Parasite Interaction. Int J Parasitol 26, 951-962.

Fiel C, P Steffan. 1994. Epidemiología de los nematodos gastrointestinales en la pampa húmeda. En: Nari A, Fiel C (eds). Enfermedades parasitarias de importancia económica en bovinos. Bases epidemiológicas para su prevención y control. Editorial Hemisferio Sur, Montevideo, Uruguay.

Gasbarre LC, JE Miller 2000. Genetics of helminth resistance. In: Axford RFE, Bishop SC, Nicholas FW, Owen JB (eds). Breeding for disease resistance in farm animals. CAB International, Wallingford, UK, Pp 129-152.

Geary TG, NC Sangster, DP Thompson. 1999. Frontiers in anthelmintic pharmacology. Vet Parasitol 84, 275-295.

Gray GD. 1997. The use of genetically resistant sheep to control nematode parasitism. Vet Parasitol 72, 345-366.

Houdijk JGM, S Athanasiadou. 2003. Direct and indirect effects of host nutrition on ruminant gastrointestinal nematodes. In: Mannetje Lt', Ramirez-Aviles L, CA Sandoval-Castro, JC Ku-Vera (eds). Matching Herbivore Nutrition to Ecosystems Biodiversity. Universidad Autónoma de Yucatán, Yucatán, México, Pp 213-236.

Hounzangbe-Adote MS, V Paolini, I Fouraste, K Moutairou, H Hoste. 2005. In vitro effects of four tropical plants on three life-cycle stages of the parasitic nematode, Haemonchus contortus. Res Vet Sci 78, 155-160. 
Jackson F, RL Coop. 2000. The development of anthelmintic resistance in sheep nematodes. Parasitology 120, 95-107.

Kuehl R. 2001. Diseño de experimentos. Principios estadísticos de diseño y análisis de investigación. $2^{\mathrm{da}}$ ed. Thomson Learning, México D.F., México.

Landau S, N Silanikove, Z Nitsan, D Barkai, H Baram, FD Provenza, A Perevolotsky. 2000. Short-term changes in eating patterns explain the effects of condensed tannins on feed intake in heifers. Appl Anim Behav Sci 69, 199-213.

Larsen M. 1999. Biological control of helminths. Int J Parasitol 29, 139-146.

Larsen M. 2000. Prospects for controlling animal parasitic nematodes by predacious micro fungi. Parasitology 120, S120-S131.

Lassak EV, T McCarthy. 1983. Australian Medicinal Plants. Methuen Australia, North Ryde, NSW, Australia.

Lyndall-Murphy M. 1993. Anthelmintic resistance in sheep. In: Corner LA, Bagust TJ (eds). Australian Standard Diagnostic Techniques. Standing Committee on Agriculture and Resource Management, CSIRO, Melbourne, Australia.

Makkar HPS. 2003. Effects and fate of tannins in ruminant animals, adaptation to tannins and strategies to overcome detrimental effects of feeding tannin-rich feeds. Small Ruminants Res 49, 241-256.

McKellar QA. 1997. Ecotoxicology and residues of anthelmintic compounds. Vet Parasitol 72, 413-435.

McLeod RS. 1995. Cost of major parasites to the Australian livestock industries. Int J Parasitol 25, 1363-1367.

Min BR, TN Barry, GT Attwood, WC McNabb. 2003. The effect of condensed tannins on the nutrition and health of ruminants fed fresh temperate forages: a review. Anim Feed Sci Tech 106, 3-19.

Molan AL, RA Alexander, IM Brookes, WC McNabb. 2000 . Effect of an extract from Sulla (Hedysarum coronarium) containing condensed tannins on the migration of three sheep gastrointestinal nematodes in vitro. Proc New Zeal Soc An 60, 21-25.

Molan AL, GC Waghorn, BR Min, WC McNabb. 2000 ${ }^{\mathrm{b}}$. The effect of condensed tannins from seven herbages on Trichostrongylus colubriformis larval migration in vitro. Folia Parasitol 47, 39-44.

Molan AL, SO Hoskin, TN Barry, WC McNabb. 2000'. Effect of condensed tannins extracted from four forages on the viability of the larvae of deer lungworms and gastrointestinal nematodes. Vet Rec 147, 44-48.

Molan AL, S Sivakumaran, PA Spencer, LP Meagher. 2004. Green tea flavan-3-ols and oligomeric proanthocyanidins inhibit the motility of infective larvae of Teladorsagia circumcincta and Trichostrongylus colubriformis in vitro. Res Vet Sci 77, 239-243.

Paolini V, JP Bergeaud, C Griez, F Prevot, P Dorchies, H Hoste. $2003^{\mathrm{a}}$. Effects of condensed tannins on goats experimentally infected with Haemonchus contortus. Vet Parasitol 113, 253-261.

Paolini V, A Frayssines, F De la Farge, P Dorchies, H Hoste. 2003 Effects of condensed tannins on established populations and on incoming larvae of Trichostrongylus colubriformis and Teladorsagia circumcincta in goats. Vet Res 34, 331-339.

Paolini V, I Fouraste, H Hoste. 2004. In vitro effects of three woody plant and sainfoin extracts on 3rd-stage larvae and adult worms of three gastrointestinal nematodes. Parasitology 129, 69-77.

Rabel B, R McGregor, PGC Douch. 1994. Improved bioassay for estimation of inhibitory effects of ovine gastrointestinal mucus and anthelmintics on nematode larval migration. Int J Parasitol 5, 671-676.

SAS Institute. 2004. Statistical Analysis Systems. Version 9.1.3. SAS Institute Inc., Cary, North Carolina, USA.

Seigler DS. 2002. Economic potencial from Western Australian Acacia species: secondary plant products. Conserv Sci W Aust 4, 109-116.

Smith WD. 1999. Prospects for vaccines of helminth parasites of grazing ruminants. Int J Parasitol 29, 17-24.

Van Soest PJ. 1982. Nutritional ecology of the ruminant. O \& B Books Inc., Corvallis, Oregon, USA.

Vercruysse J, TPM Schetters, DP Knox, P Willadsen, E Claerebout. 2007. Control of parasitic disease using vaccines: an answer to drug resistance? Rev Sci Tech off Int Epiz 26, 105-115.

Waghorn GC, WC McNabb. 2003. Consequences of plant phenolic compounds for productivity and health for ruminants. $P$ Nutr Soc 62, 383-394.

Wagland BM, WO Jones, L Hribar, T Bendixen, DLA Emery. 1992. A new simplified assay for larval migration inhibition. Int J Parasitol 22, 1183-1185.

Waller PJ, SM Thamsborg. 2004. Nematode control in 'green' ruminant production systems. Trends in Parasitol, 20, 493-497.

Wood IB, NK Amaral, K Bairden, JL Duncan, T Kassai, JB Malone, JA Pankavich, RK Reinecke, O Slocombe, SM Taylor, J Vercruysse. 1995. World Association for the Advancement of Veterinary Parasitology (WAAVP) second edition of guidelines for evaluating the efficacy of anthelmintics in ruminants (bovine, ovine, caprine). Vet Parasitol 58, 181-213. 